\title{
Lymphomatoide Papulose Typ A: Ist weniger (Therapie) mehr?
}

\author{
Lymphomatoid papulosis Type A: Could Less Therapy be More?
}

Autoren

Institut
E. Markeeva, C. S. L. Müller, T. Vogt, A. Rösch

Klinik für Dermatologie, Venerologie und Allergologie, Universitätsklinikum des Saarlandes, Homburg/Saar
Bibliografie

DOI http://dx.doi.org/ $10.1055 / \mathrm{s}-0030-1256107$ Akt Dermatol 2011; 37: 19-21 ๑) Georg Thieme Verlag KG Stuttgart · New York ISSN 0340-2541

\section{Korrespondenzadresse} Evgenia Markeeva

Klinik für Dermatologie, Venerologie und Allergologie Universitätsklinikum des Saarlandes Kirrbergerstr. 1 - Gebäude 18 66421 Homburg evgenia.markeeva@uks.eu

\section{Zusammenfassung \\ $\nabla$}

Eine 29-jährige Patientin stellte sich in unserer Klinik mit seit 1 Jahr rezidivierenden papulonodösen Hautveränderungen an den Unterarmen und Beinen vor. Histologisch zeigte sich ein buntes keilförmiges dermales Infiltrat mit Lymphozyten unterschiedlicher Größe und Morphe. Immunhistologisch fanden sich große lymphoide Zellen mit hyperchromatischen Nuklei und deutlicher Expression von CD45RO und CD30. Es wurde die Diagnose einer lymphomatoiden Papulose

Die lymphomatoide Papulose (LyP) gehört zum Kreis der CD30-positiven lymphoproliferativen Erkrankungen der Haut [1]. Sie sind nach der Mycosis fungoides (MF) die zweithäufigsten kutanen T-Zelllymphome (ca. 25\%). Die LyP präsentiert sich klinisch initial mit braun-rötlichen, z. T. oberflächlich nekrotischen Papeln. Diese befinden sich oft in unterschiedlichen Entwicklungsstadien. Nach ca. 3 bis 8 Wochen kommt es meist zur spontanen Abheilung fakultativ unter Hinterlassung varioliformer Narben. Man unterscheidet 3 histologische Typen, jedoch ohne prognostische Relevanz. Gemischtzelliger Typ (A): dermales gemischtzelliges Infiltrat, bestehend aus anaplastischen großen CD30+-lymphoiden Zellen, eosinophilen und neutrophilen Granulozyten, Histiozyten und überwiegend kleinen T-Lymphozyten. Typ (B) ähnelt dem Plaque-Stadium der MF. Der anaplastische Typ (C) dagegen entspricht histomorphologisch dem kutanen CD30+ anaplastischen großzelligen T-Zell-Lymphom (PCALCL). Ungefähr 10-20\% der LyP-Patienten erkranken an weiteren lymphoproliferativen Erkrankungen wie MF, PCALCL und M. Hodgkin [2]. Interessanterweise gibt es verschiedene Verläufe: zeitgleiche Koinzidenz, sekundäres Auftreten einer LyP nach Erstdiagnose eines anderen Lymphoms, dann oft nach erfolgreicher Chemotherapie, aber
Typ A gestellt. Eine systemische Beteiligung wurde ausgeschlossen. Wir begannen eine Therapie lediglich mit topischen Kortikosteroiden mittlerer Stärke. Von einer PUVA-Therapie oder der Gabe von Methotrexat wurde, auch auf Wunsch der Patientin, abgesehen. Der Verlauf war spontan-remissiv und benigne unter dieser „Waitand-see“-Strategie. Wir diskutieren hier die relative Benignität der Erkrankung und beleuchten kritisch das Risiko klonaler Selektion potentiell aggressiverer T-Zellklone durch zu unkritischen, verfrühten Einsatz von Methotrexat bzw. PUVA.

auch Progress einer LyP in ein maligneres Zweitlymphom mit deutlicher Verschlechterung der Prognose. Es gibt experimentelle Belege dafür, dass für alle Manifestationen ein und derselbe TZellklon verantwortlich sein kann [2].

$\mathrm{Zu}$ den in erster Linie empfohlenen therapeutischen Optionen gehören - je nach Ausdehnung des Befundes - neben der bloßen Beobachtung die Exzision der Hautveränderungen, die topische Therapie mit Kortikosteroiden und die PUVA-Therapie. Systemisch ist die Behandlung mit Methotrexat in niedriger Dosierung oder als Secondline-Therapie Interferon-alpha bzw. Bexaroten möglich $[3,4]$.

\section{Kasuistik \\ $\nabla$}

Anamnese: Eine 29-jährige Patientin stellte sich in unserer Klinik mit seit ca. 1 Jahr rezidivierend auftretenden Knötchen an den Unterarmen und Beinen vor. Die Hautveränderungen würden innerhalb von 1 bis 2 Monaten spontan abheilen. Ferner klagte sie über immer wieder auftretenden Juckreiz. Vorerkrankungen waren nicht bekannt.

Befund: Es fanden sich an den Oberarmen und Beinen mehrere wenige Millimeter messende, gruppiert stehende, rötliche Papeln unterschied- 
licher Entwicklungsstadien (frische Papeln, krustöse Ulzerationen und kleine eingesunkene Närbchen) ( $\bullet$ Abb. 1).

Labor: Kein pathologischer Befund. Unauffällige T-Zell-Subpopulationen, kein Nachweis von Sezary-Zellen.

Histologie: Es imponiert ein keilförmiges dermales Infiltrat aus meist kleinen Lymphozyten mit eingestreuten großen lymphoiden Zellen mit hyperchromatischen Nuklei. Immer wieder sind einzelne eosinophile Granulozyten darstellbar. Immunphänotypisch zeigt sich eine deutliche Expression von CD45RO und verstreut im Infiltrat auch CD30, betont in den großen lymphoiden Zellen. Es waren keine B-Zell-Marker nachweisbar ( $\bullet$ Abb. 2).

Bildgebung: In der Lymphknotensonografie, der Röntgenuntersuchung des Thorax und der Abdomensonografie fanden sich keine Hinweise auf eine systemische Beteiligung bzw. Lymphom-verdächtige Raumforderungen. Auf eine Knochenmarkuntersuchung wurde im Einvernehmen mit der Patientin aktuell verzichtet.

Es wurde die Diagnose einer lymphomatoiden Papulose Typ A gestellt. Wir begannen eine topische Therapie mit mittelstarken Steroiden (Klasse II und III), teils unter Okklusion. Dadurch kam es zur beschleunigten Abheilung der Papeln unter Ausbleiben der nekrotisierenden Exulzeration und Narbenbildung. Im weiteren Verlauf wurden erneut Effloreszenzen beobachtet, die jedoch aufgrund der zeitnahen proaktiven Steroidapplikation stets in einer geringeren Ausprägung auftraten und gut kontrollierbar waren.

\section{Diskussion}

$\nabla$

Die Therapie der lymphomatoiden Papulose richtet sich in erster Linie nach der klinischen Ausprägung des Krankheitsbildes [1,5]. Alle derzeit verfügbaren Therapiemodalitäten werden eingesetzt unter dem Aspekt der Krankheitskontrolle, nicht aber der Heilung. Keine der verfügbaren Therapien beeinflusst wesentlich den natürlichen Verlauf der Erkrankung [6]. Deswegen sollte bei dieser Entität und ihrem relativ jungen Patientengut ganz besonders das Nutzen-Risikoprofil der unterschiedlichen Behandlungsoptionen abgewogen werden [7].

Zweifelsohne müssen bei einer schweren multifokalen Ausbreitung der LyP mit Gefahr eines Systembefalls adäquate Therapiemaßnahmen ergriffen werden, zu denen laut gültiger Leitlinien PUVA, Methotrexat und, weniger gut untersucht, auch Interferonalpha und Bexaroten gehören, gegebenenfalls auch unter Inkaufnahme möglicher Nebenwirkungen. Einfache solitäre oder streng lokalisierte Herde können bekanntermaßen beobachtet, mit Steroiden behandelt oder exzidiert werden [3]. Anhand dieses Fallberichts möchten wir jedoch auf die Grenzfälle eingehen, bei welchen die klinisch-therapeutische Entscheidung gerade nicht so eindeutig ist und dann oft zugunsten eines voreilig aktiven Vorgehens ausfällt, insbesondere wenn Nicht-Dermatologen und NichtDermatopathologen bei der Diagnoseetablierung mitwirken.

Die PUVA-Therapie gehört zu den Behandlungsformen mit gut untersuchter Wirksamkeit und wird daher häufig als Therapie der ersten Wahl bei multifokalen LyP-Infiltraten verwendet [8-11]. Jedoch sollte man sich auch hier immer die Risiken einer erhöhten UV-Exposition in Erinnerung rufen. Insbesondere bei Anwendung in jungen Lebensjahren können kumulative UV-Schäden inklusive Induktion epithelialer und melanozytärer Neoplasien auftreten [12-14]. Noch relevanter könnte sein, dass UV-A-Therapien durchaus neben Apoptose in den Zielzellen vor allem auch verzögert genetische Schäden induzieren können, d.h. Mutationen, Einzel- und Doppelstrangbrüche etc. [15]. Dies korrespondiert be-

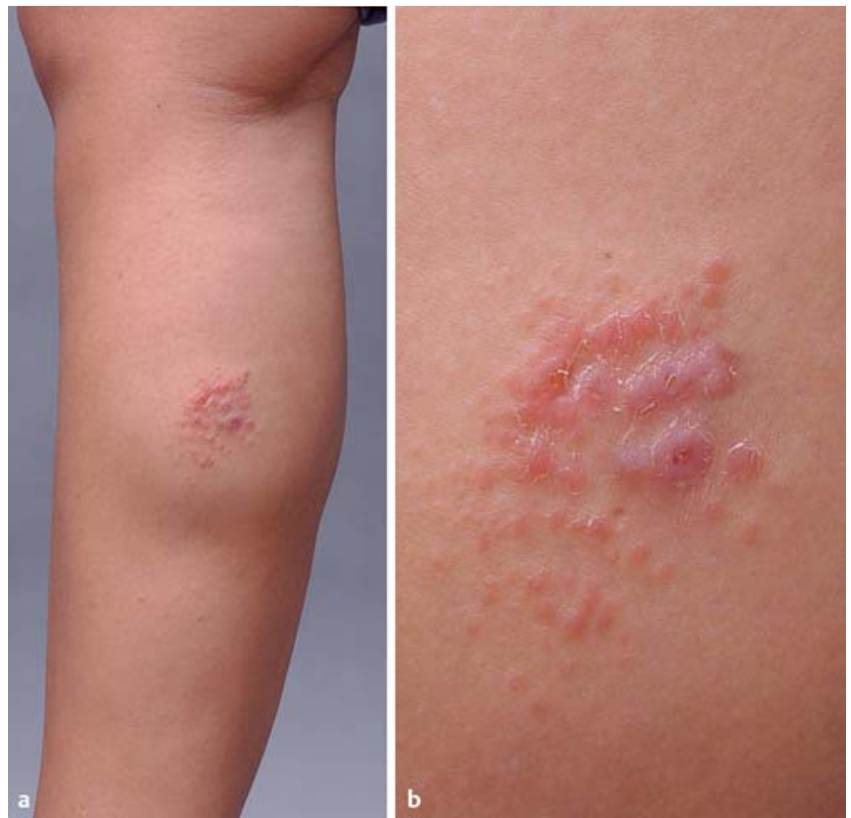

Abb. 1 Gruppiert stehende erythematöse papulo-nodöse Effloreszenzen am Unterschenkel der Patientin.

denklich mit der These, dass der Progress bei CD30+-kutanen lymphoproliferativen Erkrankungen pathobiologisch wesentlich durch klonale Selektion mit fortschreitender Akkumulation von genomischen Schäden, chromosomalen Mutationen und Mutationen auf Einzelgenebene vorangetrieben wird, wie u.a. am Beispiel des Übergangs einer LyP zu einem ALCL molekulargenetisch gezeigt wurde [2]. Eine weitere, gern verwendete Therapieoption bei multifokalem, aber oft immer noch blandem Verlauf der LyP ist die Behandlung mit niedrigdosiertem Methotrexat $[3,5,16]$. Das akute Nebenwirkungsspektrum hält sich bei der Dosierung von maximal $20 \mathrm{mg}$ pro Woche meistens noch in vertretbaren Grenzen. Es reicht unter anderem von reversiblem Haarausfall bis hin zu Blutbildveränderungen wie Leukopenie, Thrombozytopenie, Agranulozytose und Leberzellschädigungen. Allerdings mag es noch ein anderes, gravierenderes Risiko geben, welches weniger gut bekannt ist, die therapieinduzierten lymphoproliferativen Erkrankungen. Hierbei handelt es sich um die Entstehung maligner Lymphozytenklone unter einer immunosuppressiven Langzeittherapie. Fälle von B-Zell-Lymphomen (z. B. Mantelzell-Lymphom [17]) und T-Zell-Lymphomen (pannikulitisches T-Zell-Lymphom [18]) auf dem Boden zumeist chronisch rheumatischer Erkrankungen, welche über lange Zeit mit Methotrexat behandelt wurden, sind gehäuft in der Literatur zu finden $[19,20]$. Als pathogenetischer Mechanismus wird die Reaktivierung latenter EBVInfektionen diskutiert, die möglicherweise zur malignen Transformation einzelner Lymphozytenklone führt [21]. Unser Plädoyer gilt daher gerade bei der LyP mit ihrem zwar rezidivierenden, aber grundsätzlich in bis zu 90\% günstigen natürlichen Verlauf einer zurückhaltenden Therapiestrategie. Aggressivere Chemotherapien sollten auf jeden Fall unterbleiben, da sich die LyP dadurch nicht wesentlich beeinflussen lässt und sogar gehäuft nach erfolgreicher Chemotherapie anderer Lymphome beobachtet wird [2]. Interessant wäre es, epidemiologisch zu untersuchen, ob der bei LyP-Patienten beschriebene Übergang oder die Assoziation in ein Zweitlymphomen eventuell sogar durch bestimmte Primärtherapien begünstigt werden könnte. 


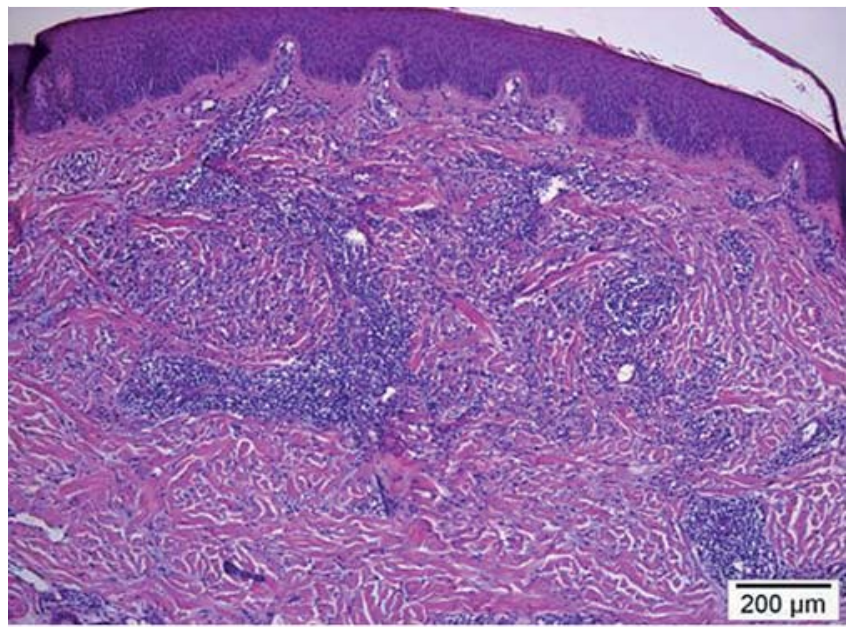

icant clinical improvement. Respecting the will of the patient, we did not start PUVA or methotrexate therapy. The course was benign and spontaneously remissive under this wait-and-see-strategy. Here we discuss the potential risks of selecting more aggressive clones by uncritical early use of methotrexate or PUVA in lymphomatoid papulosis.

\section{Literatur}

1 Burg G et al. WHO/EORTC classification of cutaneous lymphomas 2005: histological and molecular aspects. J Cutan Pathol 2005; 32 (10): 647-674

2 Kadin ME. Pathobiology of CD30+-cutaneous T-cell lymphomas. J Cutan Pathol 2006; 33: 10-17

3 Dummer R, Stadler R, Sterry W. Deutsche Leitlinie: Kutane Lymphome. In: Garbe C Hrsg. Interdisziplinäre Leitlinien zur Diagnostik und Behandlung von Hauttumoren. Stuttgart, New York: Georg Thieme; 2005: 83-95

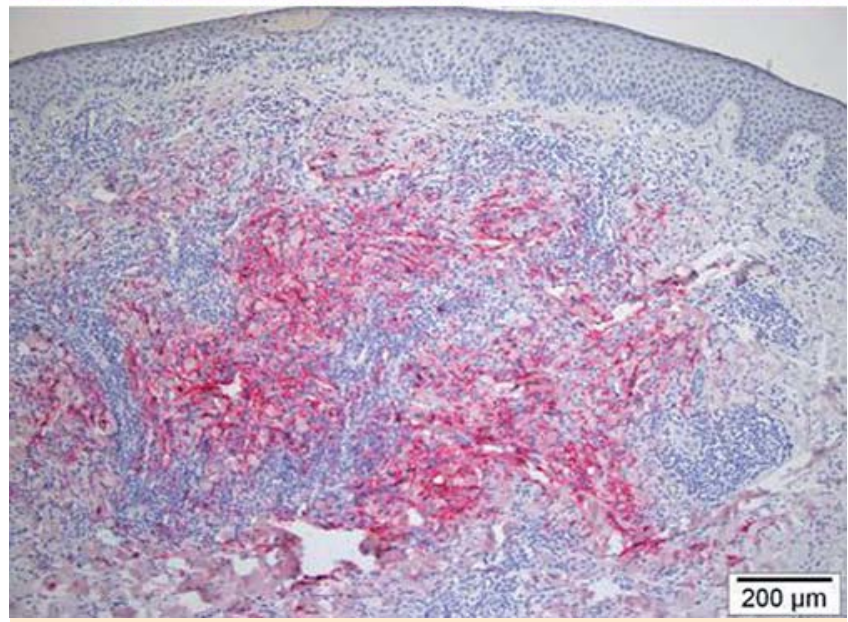

Abb. 2 Dermales Infiltrat aus großen lymphomatoiden Zellen und kleinen Lymphozyten (oben). Große lymphoide Zellen mit deutlicher Expression von CD30 (unten).

In unserem Fall haben wir aufgrund des Alters, der moderaten Ausprägung der Hauteffloreszenzen und des geringen Leidensdrucks der Patientin auch auf Wunsch der Patientin nach eingehender Beratung und Aufklärung auf die Therapie mit PUVA oder Methotrexat verzichtet. Die Patientin wurde im Umgang mit topischen Kortikosteroiden geschult. Um einen (unwahrscheinlichen) Krankheitsprogress nicht zu verpassen, führen wir engmaschige klinische Nachsorgen durch (drei- bis sechsmonatlich). Die weiterführende Labor- und bildgebende Diagnostik soll an der Klinik orientiert individualisiert erfolgen.

\section{Abstract}

\section{Lymphomatoid papulosis Type A: Could Less Therapy be More?}

A 29 year old female patient presented with papular-nodular skin lesions on her lower arms and legs that had recurred for one year. A subsequent skin biopsy showed a variegated wedge-shaped dermal infiltrate, composed of lymphocytes of different size and morphology. The immunohistochemical staining revealed large lymphoid cells with hyperchrome nuclei and an intense staining for CD30. We diagnosed lymphomatoid papulosis type A. A systemic involvement of organs was excluded. The treatment of the patient with topical class II-III corticosteroids resulted in a signif-
$4 \mathrm{Kim}$ YH et al. TNM classification system for primary cutaneous lymphomas other than mycosis fungoides and Sezary syndrome: a proposal of the International Society for Cutaneous Lymphomas (ISCL) and the Cutaneous Lymphoma Task Force of the European Organization of Research and Treatment of Cancer (EORTC). Blood 2007; 110 (2): 479 484

5 Demierre MF, Goldberg L, Kadin ME, Koh HK. Is it lymphoma or lymphomatoid papulosis? J Am Acad Dermatol 1997; 36: 765

6 Willemze R, Beljaards RC. Spectrum of primary cutaneous CD30 (Ki-1)positive lymphoproliferative disorders. A proposal for classification and guidelines for management and treatment. J Am Acad Dermatol 1993; 28 (6): 973 - 980

7 Bekkenk MW et al. Primary and secondary cutaneous CD30(+) lymphoproliferative disorders: a report from the Dutch Cutaneous Lymphoma Group on the long-term follow-up data of 219 patients and guidelines for diagnosis and treatment. Blood 2000; 95 (12): 3653 - 3661

8 Wantzin GL, Thomsen K. PUVA-treatment in lymphomatoid papulosis. Br J Dermatol 1982; 107 (6): 687-690

9 Volkenandt $M$ et al. PUVA-bath photochemotherapy resulting in rapid clearance of lymphomatoid papulosis in a child. Arch Dermatol 1995 131 (9): 1094

10 Gambichler T, Maushagen E, Menzel S. Foil bath PUVA in lymphomatoid papulosis. J Eur Acad Dermatol Venereol 1999; 13 (1): 63 - 65

11 Blondeel A et al. Lymphomatoid papulosis improved with PUVA therapy. Dermatologica 1982; 165 (5): 466 - 468

12 McGinnis KS et al. Psoralen plus long-wave UV-A (PUVA) and bexarotene therapy: An effective and synergistic combined adjunct to therapy for patients with advanced cutaneous T-cell lymphoma. Arch Dermatol 2003; 139 (6): 771 - 775

13 Dunnick JK et al. Tumors of the skin in the HRA/Skh mouse after treatment with 8-methoxypsoralen and UVA radiation. Fundam Appl Toxicol 1991; 16 (1): $92-102$

14 Stern RS, Lunder EJ. Risk of squamous cell carcinoma and methoxsalen (psoralen) and UV-A radiation (PUVA). A meta-analysis. Arch Dermatol 1998; 134 (12): $1582-1585$

15 Dahle J, Kvam E. Induction of delayed mutations and chromosomal instability in fibroblasts after UVA-, UVB-, and X-radiation. Cancer Res 2003; 63 (7): $1464-1469$

16 Yazawa $N$ et al. Successful treatment of a patient with lymphomatoid papulosis by methotrexate. J Dermatol 2001; 28 (7): 373-378

17 Tran $\mathrm{H}$ et al. Methotrexate-associated mantle-cell lymphoma in an elderly man with myasthenia gravis. Nat Clin Pract Oncol 2008; 5 (4): $234-238$

18 Nemoto Y et al. Epstein-Barr virus-infected subcutaneous panniculitislike T-cell lymphoma associated with methotrexate treatment. Int J Hematol 2010; 92 (2): 364-368

19 Clarke LE et al. Methotrexate-associated lymphoproliferative disorder in a patient with rheumatoid arthritis presenting in the skin. J Am Acad Dermatol 2007; 56 (4): 686-690

20 Huwait $H$ et al. Composite cutaneous lymphoma in a patient with rheumatoid arthritis treated with methotrexate. Am J Dermatopathol 2010; 32 (1): $65-70$

21 Feng $W H$ et al. Reactivation of latent Epstein-Barr virus by methotrexate: a potential contributor to methotrexate-associated lymphomas. J Natl Cancer Inst 2004; 96 (22): 1691 - 1702 